\title{
Lon Protease Is Involved in RhpRS-Mediated Regulation of Type III Secretion in Pseudomonas syringae
}

\author{
Tianhong Zhou, Chunyan Yin, Yingchao Zhang, Heng Shi, Jingru Wang, Linbo Sun, Xiaolong Shao, \\ Ruxia Gao, Wei Wang, and Xin Deng
}

Key Laboratory of Molecular Microbiology and Technology, Ministry of Education, TEDA Institute of Biological Sciences and Biotechnology, Nankai University, 23 Hongda Street, Tianjin 300457, China

Accepted 19 September 2016.

\begin{abstract}
Pseudomonas syringae depends on the type III secretion system (T3SS) to directly translocate effectors into host cells. Previously, we reported a nonpathogenic $\operatorname{rhpS}$ mutant, suggesting that the two-component transduction system rhpRS is an important regulator of T3SS in $P$. syringae. rhpRS regulates itself and a variety of downstream genes under an inverted repeat element promoter in a phosphorylation-dependent manner. Here, we identify lon as a suppressor of the $\operatorname{rhpS}$ mutant through transposon screening. A lon/rhpS double mutant restored the phenotypes of the $r h p S$ mutant. The expression level of lon was higher in $r h p S$ and other T3SS-deficient mutants than the wild-type strain, suggesting a negative feedback mechanism between lon and T3SS genes. lon was also induced by a novel T3SS inhibitor, acetate, which substantially compromises the activation of T3SS genes in minimal medium and bacterial growth in host plants.
\end{abstract}

Pseudomonas syringae relies on the type III secretion system (T3SS) for the successful infection in host plants (Jin et al. 2003). The T3SS genes (T3 genes) are located in the hrp/hrc (hypersensitive response and pathogenicity/hrp conserved) locus that accounts for disease symptoms in susceptible plants and the cell death-like hypersensitive response in resistant plants (Collmer et al. 2000).

In $P$. syringae, the T3 genes are inhibited in rich media, including King's B (KB) medium (King et al. 1954), and are highly activated upon colonization in the plant or in a nutrientdepleted minimal medium (MM) (Tang et al. 2006). An alternate sigma factor $\mathrm{HrpL}$ activates most $\mathrm{T} 3$ genes by directly recognizing an $h r p$ box (KGGARCY-N ${ }_{15 / 16}$-CCACNNA) in the promoters (Lam et al. 2014; Xiao and Hutcheson 1994). The $h r p L$-mediated induction of $\mathrm{T} 3$ genes requires another alternate sigma factor, RpoN $\left(\sigma^{54}\right)$, and a dimer consisting of two orthogonal transcription activators, HrpR as well as HrpS (Hendrickson et al. 2000; Hutcheson et al. 2001; Xiao and Hutcheson 1994). HrpR and HrpS form a dimer and coinduce the RpoN-dependent $h r p L$ promoter (Hutcheson et al. 2001; Jovanovic et al. 2011). A negative regulator of the T3 genes, $\mathrm{HrpV}$, directly binds and represses the activity of HrpS, which is reversed by an antirepressor, HrpG, by removing HrpV from HrpS (Preston et al. 1998; Wei et al. 2005).

An ATP-dependent protease Lon specifically targets and degrades HrpR (Bretz et al. 2002). Compared with the wild-

T. Zhou and C. Yin contributed equally to this work.

Corresponding authors: X. Deng; E-mail: dengxin@nankai.edu.cn and W. Wang; E-mail: nkweiwang@nankai.edu.cn

○ 2016 The American Phytopathological Society type (WT) strain, a lon mutant secretes more T3SS effectors, which indicates that Lon also degrades T3SS effectors. On the other hand, Lon does not degrade HrpS protein. Interestingly, the transcription of $h r p L$ has a dynamic pattern in MM in the lon mutant. The mRNA level of hrpL in the lon mutant is higher than that in the WT strain at the early time points in MM but is lower than the WT bacteria after several hours (Lan et al. 2007).

The hrpRS operon is controlled by two two-component systems (GacAS and RhpRS) (Chatterjee et al. 2003; Xiao et al. 2007). A gacA mutant displays significantly compromised expression of hrpRS and hrpL (Chatterjee et al. 2003). Compared with the WT strain, an $\operatorname{rhpS}$ (encoding a histidine kinase) mutant shows abolished T3 gene activation in the plant and MM, suggesting that RhpRS is a key two-component system (TCS) in regulating the T3SS of $P$. syringae (Deng et al. 2009; Xiao et al. 2007). However, deletion of the cognate $r h p R$ gene (encoding a response regulator) in the $r h p S$ mutant completely restores $h r p R S$ induction in MM, which indicates that RhpR is a negative regulator of $h r p R S$. RhpR regulates itself and other downstream genes carrying an inverted repeat motif (GTATC- $\mathrm{N}_{6}$-GATAC) in a phosphorylation-dependent manner (Deng et al. 2010). We later performed a genome-wide chromatin immunoprecipitation (ChIP)sequence analysis and detected 167 targets in the chromosome. The $h r p R$ promoter is one of the loci bound by $R h p R$, which demonstrates the RhpRS-mediated direct regulation of the hrpRS$h r p L$-T3SS cascade. A microarray analysis showed that RhpRS regulates more than 700 genes, consisting of more than $10 \%$ of the $P$. syringae genome. Based on our previous studies, RhpRS is an important TCS that tunes T3SS and many biological processes in different growth conditions (Deng et al. 2014; Zhou et al. 2015).

To further dissect the pathway that $r h p R S$ uses to regulate the T3SS, we conducted a suppressor screen in the rhpS background to search for mutants that are able to restore the activation of avrPto-luc in MM. These suppressors would most likely harbor mutations in genes that function downstream of rhpS. Here, we report the isolation and characterization of lon as a suppressor of the $\operatorname{rhpS}$ mutant.

\section{RESULTS}

A transposon mutagenesis screen led

to the identification of two suppressors of the $\operatorname{rhpS}$ mutant.

To further dissect the $r h p R S$ pathway, we tried to identify the postulated suppressors of the rhpS mutant. The rhpS mutant carrying avrPto-luc was subjected to EZ::TN<Tet-1> transposon insertion mutagenesis. After a 6-h induction in MM, 15,000 double mutants were screened for restored luciferase (LUC) activity. In all, five double mutants were recovered from the screen (Fig. 1A). 
To identify the suppressor genes of the $\operatorname{rhpS}$ mutant, a semidegenerate polymerase chain reaction (PCR) was used to determine the sites of the secondary transposon insertions (Jacobs et al. 2003), before the neighbor sequences of the transposon were blasted against the $P$. syringae pv. phaseolicola NPS3121 genomic sequence (Joardar et al. 2005). Three of five EZ:: $\mathrm{TN}<$ Tet-1 $>$ transposon insertions were found to be independent insertions within $r h p R$, which is expected, given the previous observations that the deletion of the $\operatorname{rhpRS}$ locus shows the same level of T3 gene induction and pathogenicity as the WT strain (Xiao et al. 2007). The other two suppressor mutants were found to be insertions in the middle of the lon gene (insertions at 309 and 687 nucleotides [nt], respectively), which encodes a Lon protease that degrades various proteins in many pathways. Both mutants showed similar phenotypes, and we used the mutant carrying an insertion at $309 \mathrm{nt}$ in the following experiments.

\section{The lon/rhpS double mutant}

displayed the phenotype of the lon mutant.

To confirm the effects of the lon/rhpS double mutations on the expression of endogenous T3 genes and to rule out mutations of the avrPto-luc reporter gene, we used a Northern analysis to examine the RNA levels of $h r p R$ and $h r p L$ in the WT P. syringae pv. phaseolicola NPS3121, rhpS mutant, lon mutant,

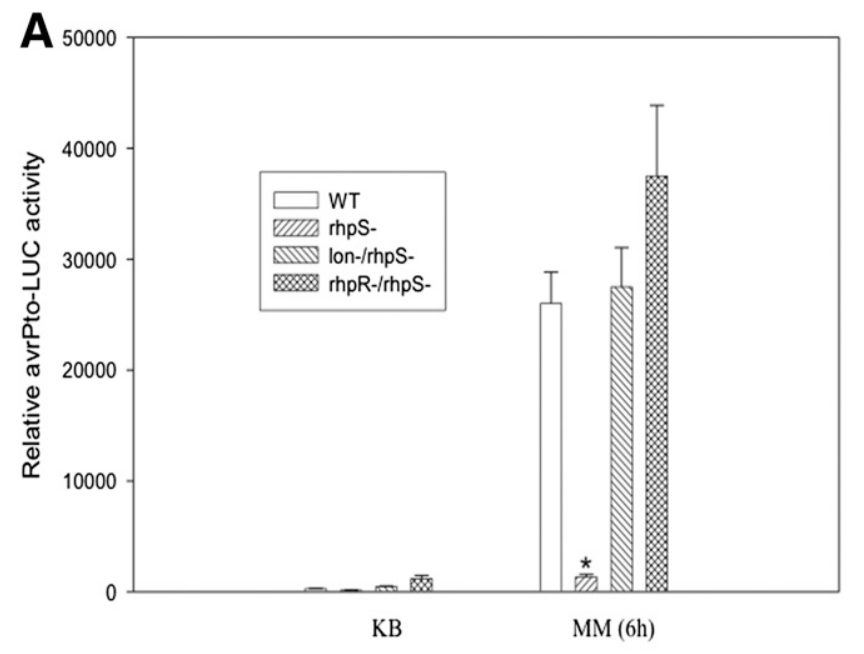

B

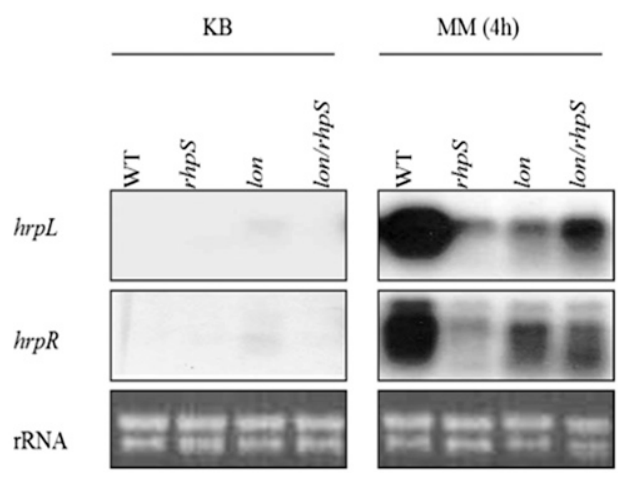

Fig. 1. A, Identification of $r h p R$ and lon as suppressors of the $r h p S$ mutant. Bacteria were grown in King's B $(\mathrm{KB})$ media and induced in minimal medium (MM) for $6 \mathrm{~h}$. The avrPto-luc activities were measured with a charge-coupled device camera. This experiment was repeated three times. An asterisk (*) denotes $P<0.05$, compared with wild type (WT) by Student's $t$ test. B, The WT (Pseudomonas syringae pv. phaseolicola) NPS3121, the rhpS mutant, the lon mutant, and the lon/rhpS double mutant strains were grown in $\mathrm{KB}$ and were induced in MM for $6 \mathrm{~h}$. Total RNA from each sample $(10 \mu \mathrm{g})$ was subjected to Northern blot analysis, which was hybridized with $h r p L$ or $h r p R$ probes. Equal loading of RNA samples is shown by the presence of similar amount of ribosomal RNA. and lon/rhpS double mutant strains by Northern hybridization. Consistent with our previous results, a lon deficiency marginally increases the $h r p L$ and $h r p R$ RNA levels in KB media, indicating that lon is a negative regulator of T3SS in KB medium (Lan et al. 2007). After $4 \mathrm{~h}$ of growth in MM, the $\operatorname{rhpS}$ mutant severely reduced the levels of $h r p L$ and $h r p R$ RNA, while the lon/rhpS double mutant largely restored this defect in MM (Fig. 1B). Consistent with our previous results, the lon mutant exhibits reduced $h r p L$ expression in MM, suggesting that Lon is a positive regulator of the $P$. syringae T3SS in MM (Lan et al. 2007).

These four strains (WT P. syringae pv. phaseolicola NPS3121, the $\operatorname{rhpS}$ mutant, the lon mutant, and the lon/rhpS double mutant) were then infiltrated at $2 \times 10^{4} \mathrm{CFU} / \mathrm{ml}$ into the leaves of host bean plants. After 6 days of growth in the plants, the lon mutation produced slightly fewer symptoms and showed about two- to threefold less growth than did the WT strain. The $\operatorname{rhpS}$ mutant was symptom-free and exhibited more than 10-fold less growth than did the WT bacteria (Fig. 2). Compared with the $\operatorname{rhpS}$ mutant, the lon/rhpS double mutant restored the defects in pathogenicity and bacterial growth to the levels of the lon mutant (Fig. 2B). These data confirmed that lon is a suppressor of the $\operatorname{rhpS}$ mutant.

\section{lon transcription was induced}

in the $\operatorname{rhpS}$ mutant and other T3-deficient mutants.

Given that lon is both an important regulator of the T3SS and a suppressor of the T3 gene-deficient $r h p S$ mutant, we propose that the expression of lon is altered in an rhpS mutant grown in MM. To test this possibility, Northern blots were performed on the $P$. syringae pv. phaseolicola WT strain and the $\operatorname{rhpS}$ mutant to compare their lon RNA levels. Deletion of the rhpS gene clearly increases the lon RNA levels in MM (Fig. 3A). Western blots further confirmed that there was more Lon protein (under its native promoter in the $\mathrm{pHM} 2$ vector) produced in the $\operatorname{rhpS}$ mutant than in WT bacteria (Fig. 3B). We propose that the autoactivated RhpR directly or indirectly upregulates lon expression in the rhpS mutant, which leads to increased degradation of HrpR and inactivation of T3 gene induction in MM. The expression level of Lon is higher in KB than that in MM, which is consistent with the Northern result in our previous report, suggesting that the lon promoter has a greater activity in KB than that in MM (Lan et al. 2007).

RhpR has been shown to bind to a few inverted repeat (IR)containing promoter regions of $r h p R S$-regulated genes (Deng et al. 2010, 2014). Because lon expression was induced in an rhpS mutant overexpressing $r h p R$ (Fig. 3A), we performed a ChIP assay to test if RhpR binds to the lon promoter, using the $\Delta r h p R S$ deletion strains (in $P$. syringae pv. tomato DC3000) overexpressing RhpR-HA (hemagglutinin) or RhpR-D70A-HA. We used the DC3000 strain because we could not obtain a clean deletion of rhpRS in the NPS3121 background after many attempts. RhpR-DNA complex was pulled down with an anti-HA antibody, which is followed by a quantitative (q)PCR to detect DNA containing the lon promoter in the immunocomplexes. The lon promoter showed a stronger interaction with RhpR than with RhpR-D70A, suggesting that this promoter not only interacts with RhpR but, also, depends on the phosphorylation of RhpR (Fig. 4).

We also examined lon RNA levels in other T3-deficient mutants, including the $h r p S$ and $h r p R$ mutants. Surprisingly, lon was induced in both mutants when grown in MM, as compared with the WT strain (Fig. 3A). Similar results were obtained from the $h r p L$ and gacA mutants grown in MM, which strongly suggests a negative feedback mechanism.

\section{Identification of acetate as a novel}

$P$. syringae T3SS inhibitor that induces lon.

Previously, an Arabidopsis protein, ATT1, was identified as a negative regulator of bacterial T3 genes. ATT1 is a cytochrome 
P450 monooxygenase that is involved in fatty acid biosynthesis in the pathway of cuticle development (Xiao et al. 2004). We propose that the expression of $\mathrm{T} 3$ genes is repressed by the products of ATT1, such as cutin monomers or other fatty acids. In order to test this hypothesis, a group of cutin-associated fatty acids, including oleic acid oxide (cis-9,10-epoxystearic acid), were found to be capable of repressing the avrPto-luc expression. Northern blot analysis confirmed that the addition of $1 \mathrm{mM}$ oleic acid oxide slightly suppressed $h r p L$ expression in MM (Fig. 5).

Our previous study shows that acetate induces the expression of $r h p R$ via phosphorylation of RhpR by acetyl phosphate (Deng et al. 2014), which led us to test if sodium acetate is able to inhibit T3 genes. The addition of $1 \mathrm{mM}$ NaAc to MM suppressed the expression of $h r p L$ in WT $P$. syringae pv. phaseolicola NPS3121 bacteria (Figs. 5 and 6A).

\section{Acetate can be used as an anti- $P$. syringae agent.}

The initial observation that $\mathrm{NaAc}$ was a potent $\mathrm{T} 3$ gene inhibitor in MM led us to believe that NaAc might be an option for bacterial disease control in plants. In greenhouse trials, spray applications of NaAc compromised bacterial growth of WT P. syringae pv. tomato DC3000 on tomato plants. Plants sprayed with $10 \mathrm{mM} \mathrm{NaAc}$ developed bacterial populations up to fivefold lower, compared with the water control, suggesting that acetate can be used as anti- $P$. syringae agent (Fig. 6B).

\section{DISCUSSION}

To identify additional players in the pathway used by $r h p R S$ to regulate the T3SS, a screen for suppressors of the rhpS
A WT hrpR hrpS rhpS hrpL gacA

lon

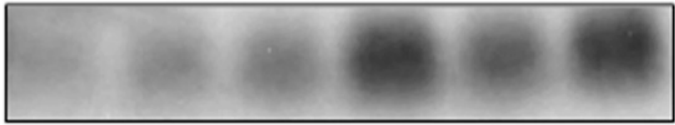

rRNA

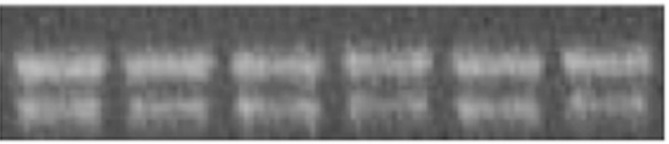

B
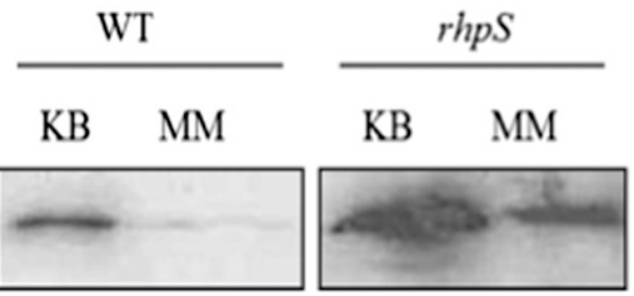

Fig. 3. A, lon expression was induced in the $r h p S$ mutant and in other type 3-deficient mutants. Wild-type (WT) Pseudomonas syringae pv. phaseolicola NPS3121, the $r h p S$ mutant, the $h r p S$ mutant, and the $h r p R$ mutant were cultured in King's B (KB) and were then induced in minimal medium (MM) for $4 \mathrm{~h}$. Total RNA from each sample $(10 \mu \mathrm{g})$ was subjected to a Northern blot, which was hybridized with the lon probe. Equal loading of RNA samples is shown by the presence of similar amount of ribosomal RNA. B, Lon-hemagglutinin (HA) protein derived from a pHM2-lon-HA reporter (native promoter) in $P$. syringae pv. phaseolicola strains. The $P$. syringae $\mathrm{pv}$. phaseolicola and rhpS mutant strains carrying a pHM2-lon-HA reporter were grown in $\mathrm{KB}$ and were induced in MM for $6 \mathrm{~h}$. Bacteria $(30 \mu \mathrm{l})$ were subjected to sodium dodecyl sulfate-polyacrylamide gel electrophoresis and Western blot analysis.
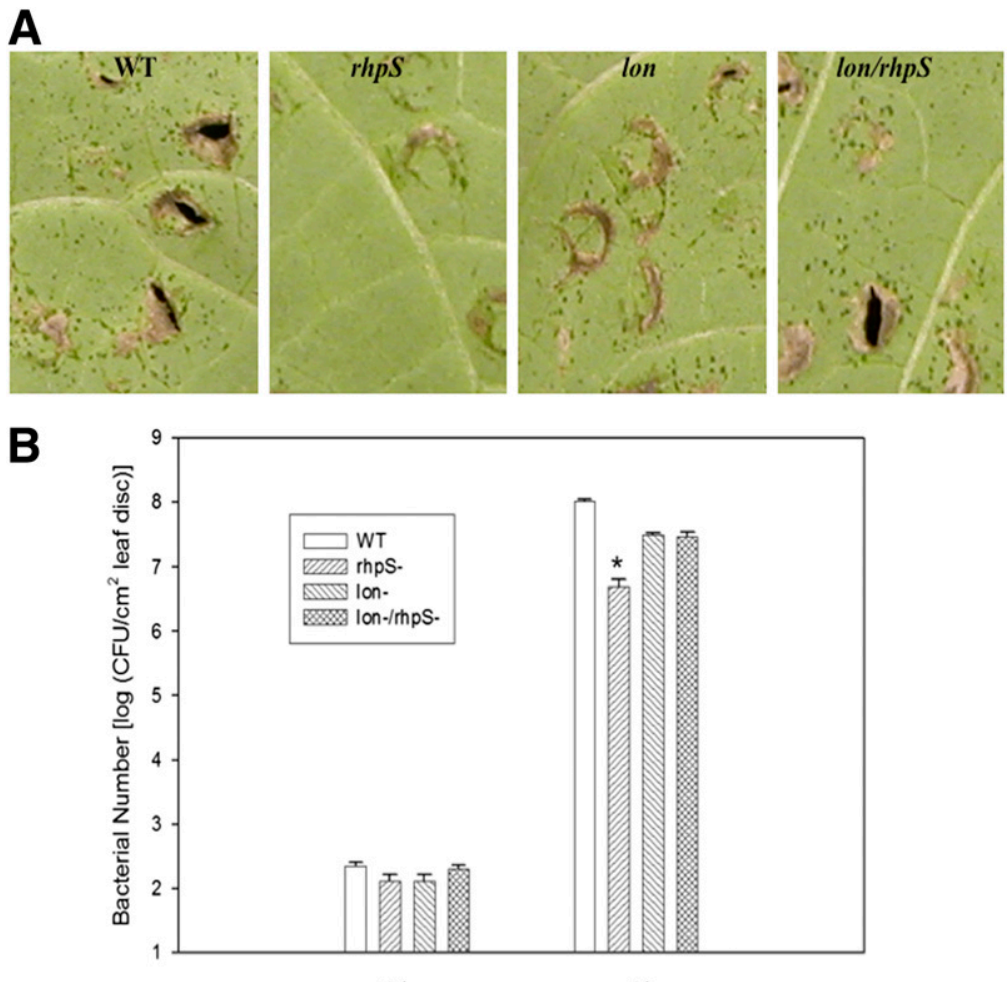

$0 \mathrm{~d}$

$6 \mathrm{~d}$

Fig. 2. A, Disease symptoms elicited by infiltrating $2 \times 10^{4} \mathrm{CFU} / \mathrm{ml}$ concentrations of wild-type (WT) Pseudomonas syringae pv. phaseolicola NPS3121, the rhpS mutant, the lon mutant, or the lon/rhpS double mutant strains into the primary leaves of 2-week-old bean plants. Pictures were photographed 5 days after inoculation. B, Bacterial number from 2-week-old bean leaves infiltrated with $2 \times 10^{4} \mathrm{CFU} / \mathrm{ml}$ oncentrations of WT P. syringae pv. phaseolicola NPS3121, the rhpS mutant, the lon mutant, or the lon/rhpS double mutant strains. For each data point, three $1-\mathrm{cm}^{2}$ leaf disks at 0 and 6 days postinoculation were homogenated and were diluted in water to count bacterial CFUs. This experiment was repeated three times. An asterisk $(*)$ denotes $P<0.05$ compared with WT by Student's $t$ test. 
mutant was performed. The rhpS-negative mutation severely abolishes avrPto-luc induction in MM, providing a clean background for the suppressor screen (Xiao et al. 2007). $r h p R$ and lon mutations completely restored avrPto-luc induction in $\mathrm{MM}$ in the rhpS background, suggesting that rhpR and lon are negative regulators of the $r h p S$-T3SS pathway. The expression level of $h r p L$ in the lon mutant was lower than that in the lon/rhpS mutant (Fig. 1B). However, the lon mutant produced pathogenicity similar to that of the $\operatorname{lon} / \mathrm{rhpS}$ mutant. There are two possible explanations. First, our previous work shows that HrpL-regulated genes exhibit different inducing patterns in the lon mutant, suggesting that additional regulators other than HrpL are involved in the regulation of T3 genes (Lan et al. 2007). Second, although there is a difference in MM, the expression level of $h r p L$ in the $r h p S$ mutant could be comparable to that in the lon/rhpS double mutant in plant. We also noticed that the RNA level of $h r p L$ was higher in WT than that in the lon mutant (Fig. 1B), but the hrpL-luc activity was similar in the two strains (Fig. 6A). This could result from the insensitivity of our charge-coupled device (CCD) camera to high luminance.

The negative role of $r h p R$ in regulating the T3SS has been reported and discussed previously (Deng et al. 2010, 2014; Xiao et al. 2007). The $\Delta r h p R S$ deletion strain showed a WT level of induction of the avrPto gene, suggesting that RhpR plays a negative role on T3 genes. In the $\operatorname{rhpS}$ mutant, RhpR is phosphorylated, binds tighter, and activates its own promoter. An IR motif (GTATC- ${ }_{6}$-GATAC) in its promoter is responsible for the RhpR-dependent induction. There are at least two other response regulators that act as suppressors to their cognate histidine kinases. A mutation of the response regulator $q s e B$ in urophathogenic Escherichia coli restores full virulence to a histidine kinase qseC knockout mutant. QseC acts as the phosphatase required for QseB dephosphorylation, which is critical for full pathogenicity (Kostakioti et al. 2009). The Salmonella two-component system pre $A B$ controls the transcription of the $\operatorname{pm} C A B$ operon. Deletion of the preA sensor but not the pre $B$ response regulator or both pre $A$ and pre $B$ results in the upregulation of $\operatorname{pmr} C A B$ (Merighi et al. 2006).

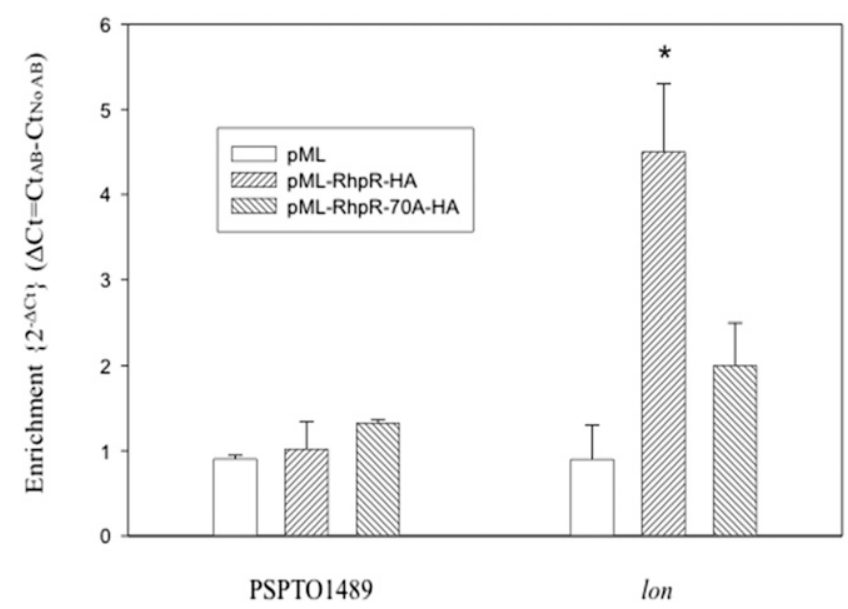

Fig. 4. Chromatin immunoprecipitation-real time-quantitative polymerase chain reaction (RT-qPCR) analysis of in vivo interaction between RhpR and the lon promoter. The lon promoter region was examined by qRT-PCR with immunoprecipitation samples of Pseudomonas syringae pv. tomato $\Delta$ rhpRS strains carrying either empty pML122, pML122-rhpR-HA, or pML122$r h p R-D 70 A-H A$. The strains were grown in King's B medium. Enrichment of lon promoter by RhpR was calculated as $2^{-\Delta \mathrm{Ct}}\left(\Delta \mathrm{Ct}=\mathrm{Ct}_{\mathrm{AB}}-\mathrm{Ct}_{\mathrm{No}} \mathrm{AB}\right)$. $\mathrm{AB}$, antibody. An asterisk (*) denotes $P<0.05$, compared with the strain containing empty vector pML by Student's $t$ test.
The restoration of virulence to the $\operatorname{rhpS}$ mutant by a second transposon insertion in lon suggests that lon is an important negative regulator of the $r h p R S$-T3SS pathway. Lon protein is a stress-induced protease that participates in a variety of biological processes by degrading a group of abnormal proteins under stringent conditions (Tsilibaris et al. 2006). Lon plays different roles in regulating T3SS in different bacteria, including directly degrading $\mathrm{HrpR}$ (Bretz et al. 2002). Lon downregulates the T3SS in Salmonella enterica serovar Typhimurium but upregulates it in Yersinia pestis. In Salmonella enterica, Lon degrades HilC and HilD, two activators of the T3SS genes (Takaya et al. 2005). However, in Yersinia pestis, Lon cleaves YmoA that negatively regulates the expression of T3 genes (Jackson et al. 2004).

Here, we propose that lon is a downstream gene of $r h p R S$. The observation that the lon'rhpS double mutant was almost indistinguishable from the lon single mutant suggests that rhpS acts with or through lon rather than in a lon-independent pathway. Compared with the WT, lon was clearly induced in the rhpS mutant when grown in MM, which suggests that enhanced lon expression in the $\operatorname{rhpS}$ mutant may account for the T3repressing phenotype. The mechanism by which lon is activated in the rhpS mutant is not clear. Even though the ChIP-real time [RT]-qPCR assay indicated that RhpR interacted with the lon promoter, we were unable to locate a highly conserved putative IR module but did locate one containing four variations (GTTTC- $\mathrm{N}_{6}$-GCTTG) in the lon promoter.

Importantly, we showed that lon expression was highly activated in multiple T3 gene-deficient mutants, strongly suggesting a feedback regulation. The lon gene of Salmonella spp. has been demonstrated to be involved in a negative feedback regulatory loop mediated by $r p o H\left(\sigma^{32}\right)$ (Matsui et al. 2008). Lon degrades HilD, the critical protein of the Salmonella T3SS regulatory loop. The expression of lon is induced by $\sigma^{32}$, which senses the cellular protein-folding environment via a negative feedback system controlled by chaperones DnaKJ and GroELS. Our previous microarray analysis indicated that $\sigma^{32}$ (PSPTO0430, rpoH) is induced threefold in an hrpRS mutant as compared with the WT strain, suggesting that $\sigma^{32}$ could be the

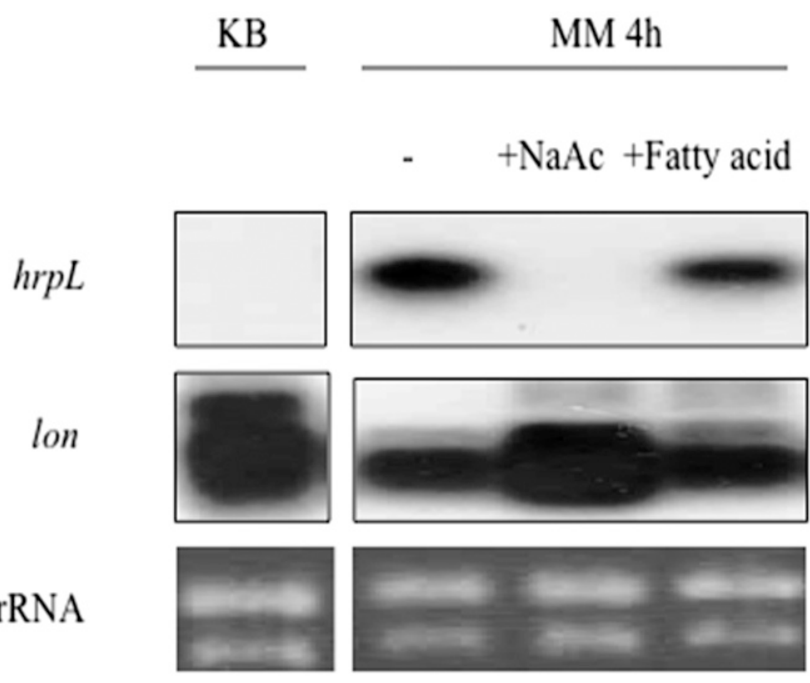

Fig. 5. Identification of acetate and a fatty acid as novel inhibitors of the type III secretion system. hrpL and lon RNAs. The wild-type Pseudomonas syringae pv. phaseolicola NPS3121 was grown in King's B and was induced in minimal medium (MM) with $1 \mathrm{mM} \mathrm{NaAc}$ or oleic acid oxide for $6 \mathrm{~h}$. Total RNA from each sample $(10 \mu \mathrm{g})$ was subjected to a Northern blot, which was hybridized with the hrpL or lon probe. Equal loading of RNA samples is shown by the presence of a similar amount of ribosomal RNA. 
regulator that is responsible for enhanced lon expression in the T3 gene-deficient mutants (Lan et al. 2007).

Lon and other heat shock proteins are induced during infection because bacteria need to adapt to various environmental stresses, such as changes in humidity and the antibacterial components from the host immune system. In Pseudomonas aeruginosa, Lon can be induced by a number of antibacterial compounds, including aminoglycosides and ciprofloxacin, which are two types of antibiotics (Brazas et al. 2007; Marr et al. 2007). Here, we showed that a novel T3 inhibitor, acetate, strongly induced lon expression. Acetate has been shown to repress the PhoPQ two-component system in E. coli in a PhoQdependent manner (Lesley and Waldburger 2003). Acetyl coenzyme A, a downstream product of the acetate metabolism pathway, inhibits the autokinase activity of PhoQ, which causes decreased PhoPQ signaling during growth in acetate. In an attempt to determine if enhanced lon expression is responsible for the inhibition of the T3SS by acetate, a hrpL-luc assay was performed to test whether the acetate-repressed LUC activity derived from $h r p L-l u c$ is abolished in the lon mutant. The lon mutant only elicited slightly higher LUC activity in MM containing acetate as compared with the WT strain, suggesting that the suppression of the T3SS by acetate largely occurs through other regulators (Fig. 6A). Acetate has been shown to be a repressor of the $p h o P Q$ system in E. coli (Lesley and Waldburger 2003). Acetic acid, a compound closely related to $\mathrm{NaAc}$,
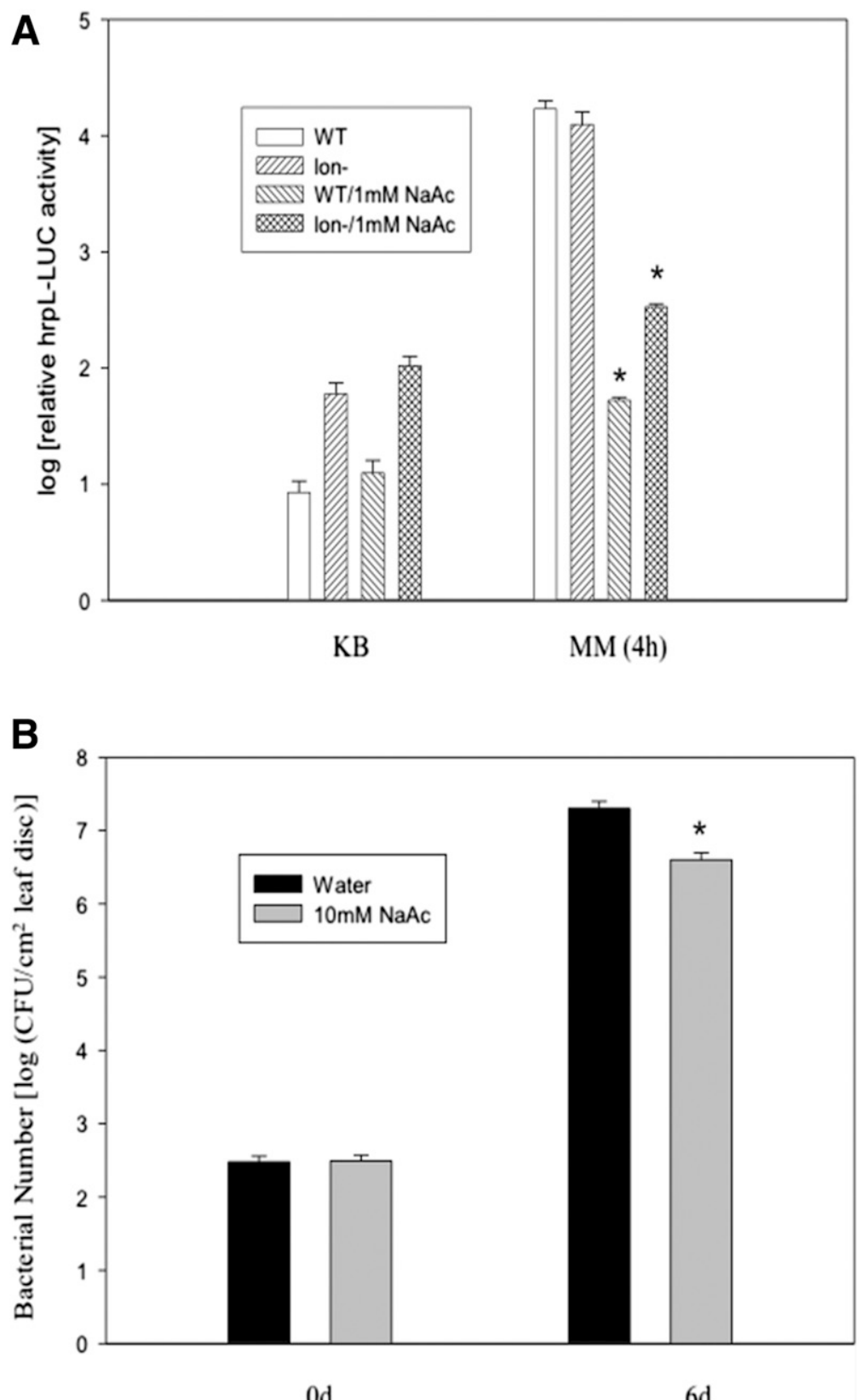

$0 \mathrm{~d}$

$6 \mathrm{~d}$

Fig. 6. A, The hrpL-luc activities in the wild-type (WT) Pseudomonas syringae pv. phaseolicola NPS3121 or the lon mutant. Bacteria were grown in minimal medium (MM) with or without $1 \mathrm{mM} \mathrm{NaAc}$ for $6 \mathrm{~h}$. The hrpL-luc activities were measured with a charge-coupled device camera. The results were repeated three times. An asterisk (*) denotes $P<0.05$, compared with their corresponding strain without NaAc treatment by Student's $t$ test. B, The WT $P$. syringae pv. tomato bacteria $\left(2 \times 10^{4} \mathrm{CFU} / \mathrm{ml}\right)$ were injected into the tomato leaves. Inoculated leaves were sprayed with water or a $10-\mathrm{mM}$ NaAc solution. Three $1-\mathrm{cm}^{2}$ leaf disks at 0 and 6 days postinoculation were homogenated and were diluted in water to count bacterial CFUs. This experiment was repeated three times. An; asterisk (*) denotes $P<0.05$ between water and NaAc treatment, by Student's $t$ test. 
has been used as an herbicide for weed control at higher concentrations $(>5 \%)$, and several acetic acid products are labeled for organic production. Although we could not rule out the possibility that $\mathrm{NaAc}$ may interfere with the physiology of tomato and then compromise bacterial growth, it seems likely that NaAc could be a candidate for organic certification.

Based on our previous and present studies on RhpRS, we propose a model of its complex regulatory roles on the T3 genes (Fig. 7). When bacteria are grown under T3 gene-inhibiting conditions (in $\mathrm{KB}$ or the $r h p S$ mutant), RhpR is phosphorylated (RhpR-P) by RhpS or acetyl phosphate. RhpR-P has higher affinity to its own promoter than RhpR, which produces more RhpR-P and directly represses $h r p R S$. RhpP-P also activates the lon promoter and produces more Lon protease that degrades HrpR. Lon positively regulates $h r p R S$ in MM. Lon is negatively

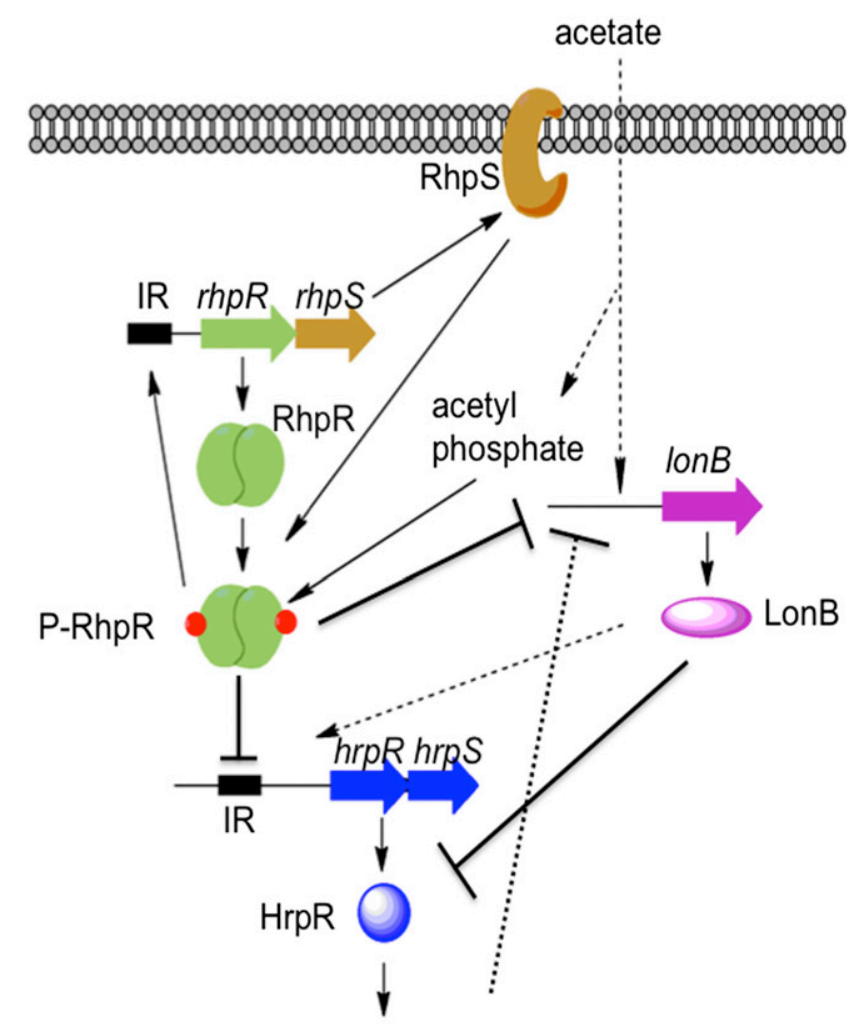

T3SS genes

Fig. 7. Proposed model of the RhpRS-Lon-type III secretion system (T3SS) regulatory network in Pseudomonas syringae. When T3SS is inhibited in King's B or the rhpS mutant, RhpR remains to be phosphorylated (RhpR-P) by RhpS or acetyl phosphate. RhpR-P binds tighter to its own promoter than unphosphorylated RhpR, which leads to higher production of RhpR-P and, thus, represses hrpRS. RhpP-P activates the lon promoter, which leads to increased production of Lon protease that specifically cuts HrpR. Lon positively regulates $h r p R S$ in minimal medium. Lon is negatively regulated by T3SS via a feedback control. Acetate induces the expression of RhpR and Lon. Acetate can be used as an anti-Pseudomonas agent. regulated by T3SS via a feedback control. Acetate also induces the expression of both T3SS negative regulators RhpR and Lon, which can be used as an anti-Pseudomonas agent.

\section{MATERIALS AND METHODS}

Plants, bacterial strains, media, and plasmids.

Bean (Phaseolus vulgaris cv. Red Kidney) and tomato (Lycopersicon esculentum cv. Rio-Grande PtoS) plants were used for virulence and bacterial growth assays in plants. The bacterial strains were the WT $P$. syringae pv. phaseolicola NPS3121 and the $\operatorname{rhpS}$, lon, lon/rhpS, hrpS, and hrpR mutants derived from the WT strain as well as the $\operatorname{rhpR} / \mathrm{rhpS}$ deletion in $P$. syringae pv. tomato DC3000. Bacteria were grown in $\mathrm{KB}$ until optical density at $600 \mathrm{~nm}\left(\mathrm{OD}_{600}\right)=2.0$. The bacteria were transferred to $\mathrm{MM}$ (Huynh et al. 1989) at $\mathrm{OD}_{600}=0.2$ and were incubated in MM for $6 \mathrm{~h}$ before extracting RNA or measuring promoter activities. The plasmids and primers are listed in Tables 1 and 2, respectively.

\section{Screen for suppressor mutants.}

The suppressor mutant library was based on the $P$. syringae pv. phaseolicola NPS3121 rhpS mutant containing the pHM2avrPto-luc reporter plasmid (Xiao et al. 2007). Colonies on KB plates with rifampicin (25 $\mathrm{mg} / \mathrm{liter})$, kanamycin $(10 \mathrm{mg} / \mathrm{liter})$, spectinomycin $(50 \mathrm{mg} / \mathrm{liter})$, and tetracycline $(10 \mathrm{mg} / \mathrm{liter})$ were transferred into fresh $100 \mu \mathrm{l}$ of $\mathrm{KB}$ in 96 -well plates and were incubated for $36 \mathrm{~h}$. The plates were centrifuged at 5, $000 \times g$ for $5 \mathrm{~min}$, and the bacteria were washed and resuspended in $500 \mu \mathrm{l}$ of MM. After $6 \mathrm{~h}, 100 \mu \mathrm{l}$ of each culture was mixed with $10 \mu \mathrm{l}$ of $0.1 \mathrm{mM}$ luciferin in a new plate. The avrPto-luc activity was quantified under a cooled CCD (Roper Scientific, Tucson, AZ, U.S.A.). Mutants showing more than fivefold avrPto-luc activities compared with the $\operatorname{rhpS}$ mutant were selected as candidate suppressor mutants.

\section{Quantitation of reporter activities.}

After being cultured in $\mathrm{KB}$ medium containing rifampicin (25 mg/liter) and spectinomycin (50 mg/liter) overnight, bacteria were washed twice and were transferred to $\mathrm{MM}\left(\mathrm{OD}_{600}=0.1\right)$. At 0 or $6 \mathrm{~h}$ after induction in MM, $100 \mu \mathrm{l}$ of culture was added with $10 \mu \mathrm{l}$ of $0.1 \mathrm{mM}$ luciferin, and the avrPto-luc activities were quantified under a cooled CCD (Roper Scientific). The data for $0 \mathrm{~h}$ in $\mathrm{MM}$ are considered as the reporter activity in KB. The CFU of bacteria were counted on KB plates. The relative avrPto-luc activities were normalized to the CFUs of bacteria.

\section{Mapping insertion sites of transposon.}

The site of specific transposon insertion of each mutant was identified by a two-stage semi-degenerate PCR, which was described by Jacobs and associates (2003). The PCR reaction uses two transposon primers (Tet1-SP1 and Tet1-SP2) and four degenerate primers (CEKG 2A, CEKG2B, CEKG 2C, and $\mathrm{CEKG})$. The resulting PCR products were sequenced with another transposon primer (Tet1-SP3). Sequences next to the

Table 1. Plasmids used in the study

\begin{tabular}{lll}
\hline Plasmid & \multicolumn{1}{c}{ Description } & Reference \\
\hline pML122-rhpR-HA & rhpR in pML122 plasmid, under pNm promoter & Xiao et al. 2007 \\
pML122-rhpR(D70A)-HA & Derived from pML122::rhpR-HA, with Asp70 replaced by Ala & Xiao et al. 2007 \\
pML122 & Broad-host plasmid & Labes et al. 1990 \\
pHM2-avrPto-luc & avrPto-luc reporter in pHM2 & Xiao et al. 2007 \\
pPTE6-hrpL-luc & hrpL-luc reporter in pPTE6 & Xiao et al. 2007 \\
pHM2-lon-HA & lon-HA in pHM2 & Lan et al. 2007 \\
\hline
\end{tabular}


insertion were blasted against the genome of $P$. syringae pv. phaseolicola NPS3121 via the National Center for Biotechnology Information.

\section{Infiltration inoculation.}

The preparation of bacteria to inoculate plants has been described previously (Shan et al. 2000). Bacteria $\left(2 \times 10^{4}\right.$ $\mathrm{CFU} / \mathrm{ml}$ ) were syringe-infiltrated into the primary leaves of bean plants ( 2 weeks old) for the virulence and bacterial growth assays. For the NaAc spray assay, either water or a $10 \mathrm{mM}$ $\mathrm{NaAc}$ solution was sprayed on the freshly inoculated tomato leaves. For the bacterial growth assays, $1-\mathrm{cm}^{2}$ leaf samples were obtained at 0 or 6 days postinoculation and were homogenated in water. The CFUs of bacteria were diluted in water and were counted in $\mathrm{KB}$ media.

\section{RNA isolation and Northern blotting.}

After being cultured in KB medium, to approximately $1 \times$ $10^{9} \mathrm{CFU} / \mathrm{ml}$, and centrifuged, bacteria were washed and were resuspended in $\mathrm{MM}\left(3 \times 10^{8} \mathrm{CFU} / \mathrm{ml}\right)$ for $6 \mathrm{~h}$. The total RNA was isolated with the RNeasy kit (Qiagen, Hilden, Germany). The primers used to amplify the DNA probes ( $h r p R$, hrpL, lon) are shown in Table 2 . The PCR products were labeled with isotope, using the Random primed DNA labeling kit (Ambion, Austin, TX, U.S.A.) to create hot probes. The following Northern blot analysis procedures were described previously (Lan et al. 2007).

\section{ChIP-qRT-PCR.}

The ChIP procedures were described previously with a ChIPIT express kit (Active Motif, Carlsbad, CA, U.S.A.) with minor changes (Bruscella et al. 2008). After being cultured overnight in $\mathrm{KB}$ media, $1 \mathrm{ml}\left(\mathrm{OD}_{600}=1.0\right)$ of Pseudomonas syringae $\mathrm{pv}$. tomato rhpRS deletion strains containing empty pML122, pML122-RhpR-HA, or pML122-RhpR-D70A-HA were crosslinked with formaldehyde and were sheared by enzyme in the ChIP-IT express kit. The following procedures were performed following the kit instructions. The resulting DNA samples were subjected to qRT-PCR assays (Bio-Rad icycler IQ; Bio-Rad, Hercules, CA, U.S.A.). The PCR primers used to amplify the lon promoter region were listed in Table 2. The SYBR green PCR mixture (Applied Biosystems, Waltham, MA, U.S.A.) was added to the ChIP samples. ChIP Enrichments were calculated by the fold change in threshold cycle $(\mathrm{Ct})$ between purified DNA with $\mathrm{HA}$ antibodies and that without HA antibodies as follows: $2^{-\Delta \mathrm{Ct}}$ $\left(\Delta \mathrm{Ct}=\mathrm{Ct}_{\mathrm{AB}}-\mathrm{Ct}_{\mathrm{No}} \mathrm{AB}\right)$. The results were repeated four times.

\section{Western blot analysis.}

Bacteria were cultured in $\mathrm{KB}$ and $\mathrm{MM}$ as mentioned above. Bacterial samples $(30 \mu \mathrm{l})$ were subjected to sodium dodecyl sulfate-polyacrylamide gel electrophoresis. The procedures of

Table 2. Primers used

\begin{tabular}{ll}
\hline Primer & \multicolumn{1}{c}{ Sequence } \\
\hline Tet1-SP1 & TGAGCGCATTGTTAGATTTC \\
Tet1-SP2 & GCTGTCAAACATGAGAATTAC \\
Tet1-SP3 & TAAGATGATCCCGGGTACC \\
CEKG 2A & GGCCACGCGTCGACTAGTACNNNNNNNNNNAGAG \\
CEKG 2B & GGCCACGCGTCGACTAGTACNNNNNNNNNNACGCC \\
CEKG 2C & GGCCACGCGTCGACTAGTACNNNNNNNNNNGATAT \\
CEKG 4 & GGCCACGCGTCGACTAGTAC \\
lon-pro195F & TTGCGTGTGAAGTGACACAA \\
lon-pro110R & GAGAAACACCACGCCAAGAT \\
hrpL-F & GACTCTTCGTCTGCCGGTAT \\
hrpL-R & GGGTCAATCTGCTGCTTCAA \\
lon-F & GATTCGTGGCCTGTACTGT \\
lon-R & TGGATATGCGTGTCGTGTTT \\
\hline
\end{tabular}

Western blot analysis were described previously (Shan et al. 2000), using the monoclonal anti-HA antibodies (Sigma, St. Louis).

\section{ACKNOWLEDGMENTS}

This work was partly supported by National Natural Science Foundation of China (31670127), the Project of Tianjin, China (13TXSYJC40100), and Tianjin Natural Science Foundation (14JCYBJC23900).

\section{LITERATURE CITED}

Brazas, M. D., Breidenstein, E. B. A., Overhage, J., and Hancock, R. E. W 2007. Role of lon, an ATP-dependent protease homolog, in resistance of Pseudomonas aeruginosa to ciprofloxacin. Antimicrob. Agents Chemother. 51:4276-4283.

Bretz, J., Losada, L., Lisboa, K., and Hutcheson, S. W. 2002. Lon protease functions as a negative regulator of type III protein secretion in Pseudomonas syringae. Mol. Microbiol. 45:397-409.

Bruscella, P., Eraso, J. M., Roh, J. H., and Kaplan, S. 2008. The use of chromatin immunoprecipitation to define PpsR binding activity in Rhodobacter sphaeroides 2.4.1. J Bacteriol. 190:6817-6828.

Chatterjee, A., Cui, Y., Yang, H., Collmer, A., Alfano, J. R., and Chatterjee, A. K. 2003. GacA, the response regulator of a two-component system, acts as a master regulator in Pseudomonas syringae pv. tomato DC3000 by controlling regulatory RNA, transcriptional activators, and alternate sigma factors. Mol. Plant-Microbe Interact. 16:1106-1117.

Collmer, A., Badel, J. L., Charkowski, A. O., Deng, W. L., Fouts, D. E., Ramos, A. R., Rehm, A. H., Anderson, D. M., Schneewind, O., van Dijk, K., and Alfano, J. R. 2000. Pseudomonas syringae Hrp type III secretion system and effector proteins. Proc. Natl. Acad. Sci. U.S.A. 97:8770-8777.

Deng, X., Lan, L., Xiao, Y., Kennelly, M., Zhou, J. M., and Tang, X. 2010. Pseudomonas syringae two-component response regulator $\mathrm{RhpR}$ regulates promoters carrying an inverted repeat element. Mol. PlantMicrobe Interact. 23:927-939.

Deng, X., Liang, H., Chen, K., He, C., Lan, L., and Tang, X. 2014. Molecular mechanisms of two-component system RhpRS regulating type III secretion system in Pseudomonas syringae. Nucleic Acids Res. 42:11472-11486.

Deng, X., Xiao, Y., Lan, L., Zhou, J. M., and Tang, X. 2009. Pseudomonas syringae pv. phaseolicola mutants compromised for type III secretion system gene induction. Mol. Plant-Microbe Interact. 22:964-976.

Hendrickson, E. L., Guevera, P., and Ausubel, F. M. 2000. The alternative sigma factor RpoN is required for hrp activity in Pseudomonas syringae pv. maculicola and acts at the level of hrpL transcription. J. Bacteriol. 182:3508-3516.

Hutcheson, S. W., Bretz, J., Sussan, T., Jin, S., and Pak, K. 2001. Enhancerbinding proteins HrpR and HrpS interact to regulate hrp-encoded type III protein secretion in Pseudomonas syringae strains. J. Bacteriol. 183: 5589-5598.

Huynh, T. V., Dahlbeck, D., and Staskawicz, B. J. 1989. Bacterial blight of soybean: Regulation of a pathogen gene determining host cultivar specificity. Science 245:1374-1377.

Jackson, M. W., Silva-Herzog, E., and Plano, G. V. 2004. The ATPdependent ClpXP and Lon proteases regulate expression of the Yersinia pestis type III secretion system via regulated proteolysis of YmoA, a small histone-like protein. Mol. Microbiol. 54:1364-1378.

Jacobs, M. A., Alwood, A., Thaipisuttikul, I., Spencer, D., Haugen, E., Ernst, S., Will, O., Kaul, R., Raymond, C., Levy, R., Chun-Rong, L., Guenthner, D., Bovee, D., Olson, M. V., and Manoil, C. 2003. Comprehensive transposon mutant library of Pseudomonas aeruginosa. Proc. Natl. Acad. Sci. U.S.A. 100:14339-14344.

Jin, Q., Thilmony, R., Zwiesler-Vollick, J., and He, S. Y. 2003. Type III protein secretion in Pseudomonas syringae. Microbes Infect. 5:301-310.

Joardar, V., Lindeberg, M., Jackson, R. W., Selengut, J., Dodson, R., Brinkac, L. M., Daugherty, S. C., Deboy, R., Durkin, A. S., Giglio, M. G., Madupu, R., Nelson, W. C., Rosovitz, M. J., Sullivan, S., Crabtree, J., Creasy, T., Davidsen, T., Haft, D. H., Zafar, N., Zhou, L., Halpin, R., Holley, T., Khouri, H., Feldblyum, T., White, O., Fraser, C. M., Chatterjee, A. K., Cartinhour, S., Schneider, D. J., Mansfield, J., Collmer, A., and Buell, C. R. 2005. Whole-genome sequence analysis of Pseudomonas syringae pv. phaseolicola 1448A reveals divergence among pathovars in genes involved in virulence and transposition. J. Bacteriol. 187:6488-6498

Jovanovic, M., James, E. H., Burrows, P. C., Rego, F. G., Buck, M., and Schumacher, J. 2011. Regulation of the co-evolved HrpR and HrpS AAA+ 
proteins required for Pseudomonas syringae pathogenicity. Nat. Commun. 2:177.

King, E. O., Ward, M. K., and Raney, D. E. 1954. Two simple media for the demonstration of pyocyanin and fluorescin. J. Lab. Clin. Med. 44: 301-307.

Kostakioti, M., Hadjifrangiskou, M., Pinkner, J. S., and Hultgren, S. J. 2009. QseC-mediated dephosphorylation of QseB is required for expression of genes associated with virulence in uropathogenic Escherichia coli. Mol. Microbiol. 73:1020-1031.

Labes, M., Puhler, A., and Simon, R. 1990. A new family of RSF1010derived expression and $l a c$-fusion broad-host-range vectors for gramnegative bacteria. Gene 89:37-46.

Lam, H. N., Chakravarthy, S., Wei, H.-L., BuiNguyen, H., Stodghill, P. V., Collmer, A., Swingle, B. M., and Cartinhour, S. W. 2014. Global analysis of the HrpL regulon in the plant pathogen Pseudomonas syringae pv. tomato DC3000 reveals new regulon members with diverse functions. PLoS One 9:e106115.

Lan, L., Deng, X., Xiao, Y., Zhou, J. M., and Tang, X. 2007. Mutation of Lon protease differentially affects the expression of Pseudomonas syringae type III secretion system genes in rich and minimal media and reduces pathogenicity. Mol. Plant-Microbe Interact. 20:682-696.

Lesley, J. A., and Waldburger, C. D. 2003. Repression of Escherichia coli PhoP-PhoQ signaling by acetate reveals a regulatory role for acetyl coenzyme A. J. Bacteriol. 185:2563-2570.

Marr, A. K., Overhage, J., Bains, M., and Hancock, R. E. W. 2007. The Lon protease of Pseudomonas aeruginosa is induced by aminoglycosides and is involved in biofilm formation and motility. Microbiology 153:474-482.

Matsui, M., Takaya, A., and Yamamoto, T. 2008. Sigma32-mediated negative regulation of Salmonella pathogenicity island 1 expression. J. Bacteriol. 190:6636-6645.

Merighi, M., Carroll-Portillo, A., Septer, A. N., Bhatiya, A., and Gunn, J. S. 2006. Role of Salmonella enterica serovar Typhimurium two-component system PreA/PreB in modulating PmrA-regulated gene transcription. J. Bacteriol. 188:141-149.
Preston, G., Deng, W. L., Huang, H. C., and Collmer, A. 1998. Negative regulation of hrp genes in Pseudomonas syringae by HrpV. J. Bacteriol. 180:4532-4537.

Shan, L., Thara, V. K., Martin, G. B., Zhou, J. M., and Tang, X. 2000. The Pseudomonas AvrPto protein is differentially recognized by tomato and tobacco and is localized to the plant plasma membrane. Plant Cell 12: 2323-2338.

Takaya, A., Kubota, Y., Isogai, E., and Yamamoto, T. 2005. Degradation of the HilC and HilD regulator proteins by ATP-dependent Lon protease leads to downregulation of Salmonella pathogenicity island 1 gene expression. Mol. Microbiol. 55:839-852.

Tang, X., Xiao, Y., and Zhou, J. M. 2006. Regulation of the type III secretion system in phytopathogenic bacteria. Mol. Plant-Microbe Interact. 19:1159-1166.

Tsilibaris, V., Maenhaut-Michel, G., and Van Melderen, L. 2006. Biological roles of the Lon ATP-dependent protease. Res. Microbiol. 157:701-713.

Wei, C. F., Deng, W. L., and Huang, H. C. 2005. A chaperone-like HrpG protein acts as a suppressor of $\mathrm{HrpV}$ in regulation of the Pseudomonas syringae pv. syringae type III secretion system. Mol. Microbiol. 57:520-536.

Xiao, F., Goodwin, S. M., Xiao, Y., Sun, Z., Baker, D., Tang, X., Jenks, M. A., and Zhou, J. M. 2004. Arabidopsis CYP86A2 represses Pseudomonas syringae type III genes and is required for cuticle development. EMBO J. 23:2903-2913.

Xiao, Y., and Hutcheson, S. W. 1994. A single promoter sequence recognized by a newly identified alternate sigma factor directs expression of pathogenicity and host range determinants in Pseudomonas syringae. J. Bacteriol. 176:3089-3091.

Xiao, Y., Lan, L., Yin, C., Deng, X., Baker, D., Zhou, J. M., and Tang, X. 2007. Two-component sensor RhpS promotes induction of Pseudomonas syringae type III secretion system by repressing negative regulator RhpR. Mol. Plant-Microbe Interact. 20:223-234.

Zhou, T., Chen, K., Zhang, H. X., and Deng, X. 2015. Genome-wide DNA binding pattern of two-component system response regulator RhpR in Pseudomonas syringae. Genom. Data 4:146-147. 